\title{
NUEVOS REGISTROS DE SOLANUM L. (SOLANACEAE) PARA EL BAJÍO Y REGIONES ADYACENTES
}

\author{
Aarón Rodríguez Contreras \\ Y \\ Ofelia Vargas Ponce \\ Departamento de Botánica y Zoología \\ Centro Universitario de Ciencias Biológicas y Agropecuarias \\ Universidad de Guadalajara \\ Apartado postal 139 \\ 45101 Zapopan, Jalisco, México
}

\section{RESUMEN}

Se presentan cinco especies de Solanum (Solanaceae) como nuevos registros para la flora del Bajío y regiones adyacentes. Solanum brachistotrichum, S. hintonii, S. jamesii y S. morelliforme son papas silvestres y se agrupan en la sección Petota. Por su parte, $S$. triquetrum pertenece a la sección Dulcamara. Se comentan los rasgos distintivos de estos taxa y se amplía su distribución conocida. Por último, se incluye la ilustración de S. triquetrum.

Palabras clave: Solanum, Solanaceae, México.

\section{ABSTRACT}

We report, for the first time, the presence of five species of Solanum for the Bajío and neighboring regions flora. Solanum brachistotrichum, S. hintonii, S. jamesii y S. morelliforme are grouped in Solanum section Petota while $S$. triquetum is part of Solanum section Dulcamara. We highlight the distinctive characteristics of each species and update their known geographical distribution. Finally, we present a drawing of $S$. triquetrum.

Key words: Solanum, Solanaceae, Mexico.

\section{INTRODUCCIÓN}

Solanum L. es el género más diversificado de la familia Solanaceae y agrupa a unas 1000 especies (D’Arcy, 1991). En México, está integrado por 150 especies (Nee, 1993). Actualmente, se trabaja en un estudio taxonómico y florístico de Solanum para la flora del Bajío y hasta ahora se han reconocido 58 especies (Rodríguez, en prep.), cinco de las cuales constituyen registros nuevos para la flora de la región. La zona de estudio se localiza en la parte central de México e incluye a los estados de Guanajuato, Querétaro y la parte 
norte de Michoacán. En esta última entidad, quedan incluidas las porciones situadas al este del meridiano $102^{\circ} 10^{\prime} \mathrm{W}$ y al norte del parteaguas de la cuenca del río Balsas (Rzedowski y Rzedowski, 1991). Los ejemplares botánicos que se estudiaron provienen de colectas recientes realizadas por los autores y del herbario del Instituto de Ecología, A.C., Centro Regional del Bajío (IEB).

\section{RESULTADOS Y DISCUSIÓN}

Solanum brachistotrichum (Bitter) Rydb., Bull. Torrey Bot. Club 51: 170. 1924.

Herbácea perenne, estolonífera y tuberífera, de $20-75 \mathrm{~cm}$ de alto, erecta 0 ascendente, pubescente o pilosa. Hojas imparipinnadas, no mayores de $10 \mathrm{~cm}$ de largo con (3-)5-7(-9) folíolos subsésiles a peciolulados, lanceolados a linear-lanceolados, foliólulos intersticiales ocasionalmente 2, ovado-lanceolados; hojas pseudoestipulares falcadas o semiovadas. Inflorescencia cimoso-paniculada, con 4-13 flores; cáliz de 2-3 mm de largo, piloso, dividido en su mitad en lóbulos triangulares u oblongos, agudos; corola estrellada, de $1.5-3 \mathrm{~cm}$ de diámetro, blanca o en ocasiones el ápice de los lóbulos teñido de color violeta, lóbulos reflejos. Fruto globoso, de $1 \mathrm{~cm}$ de diámetro, de color verde.

Solanum brachistotrichum es morfológicamente similar a S. stenophillidium Bitter. Sin embargo, estas dos especies difieren entre sí en el tamaño de la planta y forma, así como en el tamaño e indumento de la hoja. Solanum stenophillidium mide $25-80 \mathrm{~cm}$ de alto, sus hojas varían de (4-)7-25 cm de largo, con (3-)5(-7) folíolos lineares a linear-lanceolados, de $2-9 \mathrm{~cm}$ de largo y de $0.4-1.7 \mathrm{~cm}$ de ancho, glabros o minutamente pilosos. En contraste, S. brachistotricum mide $20-75 \mathrm{~cm}$ de alto, sus hojas son de $4-9 \mathrm{~cm}$ de largo, con (3-)7(-9) folíolos lanceolados a linear-lanceolados, de $2-3 \mathrm{~cm}$ de largo y de $0.5-1.5 \mathrm{~cm}$ de ancho, pilosos. Una ilustración de S. brachistotrichum fue publicada por Correll (1952: fig. 71; 1962: fig. 103), así como por Rodríguez y Vargas (1994: fig. 3).

Fenología: Florece de julio a agosto y fructifica de agosto a septiembre.

Hábitat: En el norte de México crece en el matorral xerófilo. También es común entre los cultivos de maíz y con frecuencia se le encuentra a la sombra de Mimosa monancistra y Opuntia. En Michoacán, las poblaciones conocidas de S. brachistrotrichum crecen en el encinar muy perturbado a una altitud de $1950 \mathrm{~m}$.

Distribución: Solanum brachistotrichum se conocía de los estados de Sonora, Chihuahua, Durango, Zacatecas, Aguascalientes y Jalisco (Correll, 1962; Hawkes, 1990; Rodríguez y Vargas, 1994).

Ejemplares revisados: Michoacán: autopista México-Guadalajara, entre los cruceros a La Piedad y Zacapu, 5 km antes de llegar al puente La Medina, km 319-320, 1955'N y 10200'W, municipio de Panindícuaro, A. Rodríguez et al. 2661 (CHAPA, CIIDIR-DGO, ENCB, IBUG, IEB, MEXU, PTIS, WIS, XAL); km 320 autopista de cuota México-Guadalajara, municipio de Panindícuaro, A. Rodríguez 2907 (CHAPA, ENCB, IBUG, IEB, MEXU, PTIS, WIS). 
Rodríguez y Vargas: Nuevos Registros de Solanum para el Bajío y Regiones Adyacentes

Solanum hintonii Correll, Wrightia 2: 139. 1961.

Herbácea perenne, estolonífera y tuberífera, de 40-125 cm de alto, erecta, glabra o esparcidamente pubescente. Hojas imparipinnadas, hasta de $14 \mathrm{~cm}$ de largo, con 5-7 folíolos peciolulados, lanceolados; hojas pseudoestipulares lunuladas o falcadas, sin foliólulos intersticiales. Inflorescencia cimoso-paniculada, con 12-28 flores; cáliz de $5 \mathrm{~mm}$ de largo, dividido cerca de su mitad en lóbulos ovados y acuminados; corola estrellada, hasta de $2 \mathrm{~cm}$ de diámetro, blanca, lóbulos angostos, fuertemente reflejos. Fruto cónico, de $2.2 \mathrm{~cm}$ de largo y $0.8 \mathrm{~mm}$ de ancho, de color verde.

La diagnosis de $S$. hintonii no incluye características del fruto, pero menciona un ovario elipsoide (Correll, 1961, 1962). Hawkes (1990) cita un fruto elipsoide pero no proporciona tamaño alguno. El ejemplar de herbario colectado en Querétaro (J. Rzedowski 45052) posee un fruto que fue utilizado para la descripción. De igual forma, se desconocía el tubérculo de $S$. hintonii. El material examinado y colectado en Guanajuato ( $E$. Ventura y E. López 7282) posee un tubérculo globoso, de $1.1 \mathrm{~cm}$ de diámetro.

Morfológicamente, S. hintonii es similar a S. trifidum Correll y S. x ehrenbergii (Bitter) Rydb. Solanum trifidum comparte con $S$. hintonii los frutos cónicos y corola estrellada, blanca, pero difiere por ser pubescente y por tener hojas mayormente trifolioladas, aunque puede presentar 5-7 folíolos sésiles, entonces el par basal es muy reducido en tamaño. Por otro lado, $S$. hintonii y $S$. x ehrenbergii poseen hojas con 5-7 folíolos, ovado-lanceolados, enteros o sinuados en el margen. Sin embargo, a diferencia de $S$. x ehrenbergii, S. hintonii no tiene foliólulos intersticiales. Más aún, $S$. hintonii produce fruto cónico mientras que el de $S$. x ehrenbergii es globoso. Correll (1962) provee la ilustración de S. hintonii (fig. 139).

Fenología: Florece de agosto a octubre y fructifica en octubre.

Hábitat: El material tipo de S. hintonii se localizó a lo largo de cercos de piedra a una altitud de 1700 m (Correll, 1961, 1962). En Querétaro, se encontró en suelo calizo con vegetación de bosque de Garrya y Juniperus y en Guanajuato se colectó en ladera de cerro con vegetación boscosa. Crece en un intervalo altitudinal que va de los $1700 \mathrm{~m}$ hasta los $2800 \mathrm{~m}$.

Distribución: Solanum hintonii se conocía sólo de la localidad tipo en Temascaltepec, Estado de México (Correll, 1962; Hawkes, 1990).

Ejemplares revisados: Querétaro: $5 \mathrm{~km}$ al NW de El Doctor, municipio de Cadereyta, J. Rzedowski 45052 (IEB). Guanajuato: El Charco, $25 \mathrm{~km}$ al norte de Xichú, municipio de Xichú, E. Ventura y E. López 7282 (IEB).

Solanum jamesii Torr., Ann. Lyceum Nat. Hist. New York 2: 227. 1828.

Herbácea perenne, estolonífera y tuberífera, de $30-50 \mathrm{~cm}$ de alto, erecta o extendida, glabra o pilosa, los tricomas glandulares, con un tono gris-azulado. Hojas imparipinnadas, de 2.8-5.8 cm de largo, con (5)7(13) folíolos angostamente elípticos a ovados u ovado- 
lanceolados, folíolos inferiores muy reducidos en tamaño, sin foliólulos intersticiales o éstos rara vez presentes; hojas pseudoestipulares y axilares pinnati-compuestas, pero mucho más pequeñas que las hojas. Inflorescencia cimoso-paniculada, con 3-9 flores; cáliz de $8 \mathrm{~mm}$ de largo, partido casi en su base; corola estrellada, de $1.3-3.2 \mathrm{~cm}$ de diámetro y $1.8 \mathrm{~cm}$ de largo, blanca, lóbulos ovado-lanceolados. Fruto globoso, menor de $1 \mathrm{~cm}$ de diámetro, de color verde.

Solanum jamesii se caracteriza por la presencia de hojas pseudoestipulares o axilares pinnati-compuestas, corola partida y el cáliz florífero alargado. Solanum pinnatisectum Dunal también presenta hojas pseudoestipulares o axilares pinnati-compuestas, pero difiere por tener un mayor número de folíolos, de 9-17(-27), que son linear-oblongos o angostamente elíptico-lanceolados. Una foto del tipo e ilustración de $S$. jamesii fue publicada por Correll (1952: fig. 74 y 75; 1962: fig. 104).

Fenología: Florece de julio a noviembre y fructifica de agosto a noviembre.

Hábitat: Solanum jamesii crece en suelos calizos de laderas rocosas a lo largo de cañones y cañadas, a la orilla de ríos o cerca de campos de cultivo en altitudes que casi alcanzan los 3000 m. En Querétaro, S. jamesii se colectó en el bosque de Abies, creciendo sobre rocas calizas y se informa que es muy abundante.

Distribución: Crece en el suroeste de Estados Unidos de América y México. En México, se conocía de Sonora y San Luis Potosí (Correll, 1952 y 1962; Hawkes, 1990).

Ejemplares revisados: Querétaro: cerro El Espolón, cerca del Rancho Pinalito, municipio de Cadereyta, E. Pérez y S. Zamudio 3488 (IEB).

Solanum morelliforme Bitter et Muench, Repert. Spec. Nov. Regni Veg. 12: 154. 1913.

Herbácea perenne, estolonífera y tuberífera, de 20 a $45 \mathrm{~cm}$ de alto, epífita de porte delicado, erecta o decumbente, glabra o con algunos pelos simples. Hojas simples, alternas, de 2-3.8 cm de largo, láminas ovado-lanceoladas, acuminadas; hojas pseudoestipulares lanceoladas, acuminadas, de menos de $1 \mathrm{~cm}$ de largo. Inflorescencia pseudoterminal, cimosa, con 2-15 flores; cáliz de 1.5-2 mm de largo, los lóbulos muy pequeños; corola estrellada, hasta de $0.8 \mathrm{~mm}$ de largo y $1.6 \mathrm{~cm}$ de diámetro, blanca con tonos de color violeta en el ápice de los lóbulos. Fruto globoso, de $6 \mathrm{~mm}$ de diámetro, de color verde.

Solanum morelliforme se distingue por su hábito epífito, hojas simples y corolas pequeñas estrelladas. Una ilustración de $S$. morelliforme fue publicada por Correll (1952: fig. 60; 1962: fig. 92), así como por Rodríguez y Vargas (1994: fig. 9).

Fenología: Florece de julio a agosto y fructifica en octubre.

Hábitat: Solanum morelliforme es común sobre troncos de encino en bosque mesófilo de montaña de la vertiente del Golfo de México. También se le ha encontrado sobre rocas y paredes cubiertas con musgo dentro del mismo tipo de vegetación a una altitud de 1600 a $2700 \mathrm{~m}$. 
Distribución: Solanum morelliforme crece en México y Guatemala. En México, ha sido citada de los estados de Jalisco, Michoacán, México, Puebla, Veracruz, Guerrero y Chiapas (Breedlove, 1986; Nee, 1993; Hawkes, 1990; Rodríguez y Vargas, 1994; Rodríguez-Jiménez y Espinosa-Garduño, 1996). En Michoacán, S. morelliforme se ha colectado en los alrededores de Capacuaro en el municipio de Uruapan (Flores, 1966; Rodríguez-Jiménez y Espinosa-Garduño, 1996). Esta zona representa el límite suroeste de la región de estudio. Aquí, informamos de su presencia en los estados de Querétaro e Hidalgo. El estado de Hidalgo no forma parte del Bajío pero el registro de $S$. morelliforme en esta entidad amplía su área de distribución geográfica.

Ejemplares revisados: Querétaro: Los Mixcahuales, $3 \mathrm{~km}$ al NE de La Yesca, municipio Landa de Matamoros, E. González 1124 (IEB). Michoacán: carretera Uruapan a Carapan, poco delante de Capacuaro, municipio de Uruapan, R. Flores-Crespo S-816 (CHAPA, ENCB, MEXU); vicinity of Capacuaro, D. Ugent et al. 31350 (ENCB, LL). Hidalgo: predio El Tejocote, al W de Agua Blanca de Iturbide, $2 \mathrm{~km}$ en línea recta desde el centro del pueblo, municipio de Agua Blanca de Iturbide, H. García y E. Guízar 90 (IEB).

Solanum triquetrum Cav., Icon. PI. 3: 3, tab. 259. 1795.

Herbácea erecta o semitrepadora a subfrutescente, de $25-50 \mathrm{~cm}$ de alto, glabra 0 con tricomas simples, adpresos, blancos. Hojas alternas, simples, de 1.1-4.3 cm de largo, triangular-hastadas o lanceolado-hastadas, ensanchadas en la base y atenuadas en el ápice, el margen entero, liso o con 2 ó 3 lóbulos irregulares a lo largo de la lámina. Inflorescencia cimosa, con 3-8 flores, pedicelos articulados en su base; cáliz de $1.5 \mathrm{~mm}$ de largo, dividido cerca de su mitad en lóbulos triangulares; corola estrellada, hasta de $6 \mathrm{~mm}$ de largo, blanca. Fruto una baya, hasta de $1 \mathrm{~cm}$ de diámetro, cuando inmadura apiculada, de color verde y al madurar de color rojo (Fig. 1).

Las hojas triangular-hastadas con pubescencia canescente son características que permiten reconocer a $S$. triquetrum.

Fenología: Florece en agosto y fructifica en octubre.

Hábitat: En Guanajuato, las poblaciones conocidas de $S$. triquetrum se localizaron en terrenos planos con pastizal y matorral submontano y las de Querétaro en el bosque tropical caducifolio, integrando un intervalo altitudinal de 850 a 1900 m.

Distribución: Solanum triquetrum se conoce de Estados Unidos de América y México. Para México, se ha registrado de los estados de Coahuila, Nuevo León, Durango y San Luis Potosí (Standley, 1924; Reeves y Bain, 1947; González-Elizondo et al., 1991). Las poblaciones de $S$. triquetrum que mencionamos en este artículo constituyen el límite sur, hasta ahora conocido, de la distribución geográfica de la especie. En Guanajuato, las dos poblaciones aquí citadas fueron muestreadas en la región de la Sierrra Gorda al noreste del estado. Rzedowski et al. (1996) destacan la similitud florística del noreste de Guanajuato y las zonas vecinas de los estados de Querétaro y San Luis Potosí. Precisamente, el municipio de Jalpan, Querétaro, colinda con la región noreste de Guanajuato. No es extraño, 
Acta Botanica Mexicana (2001), 56: 1-8
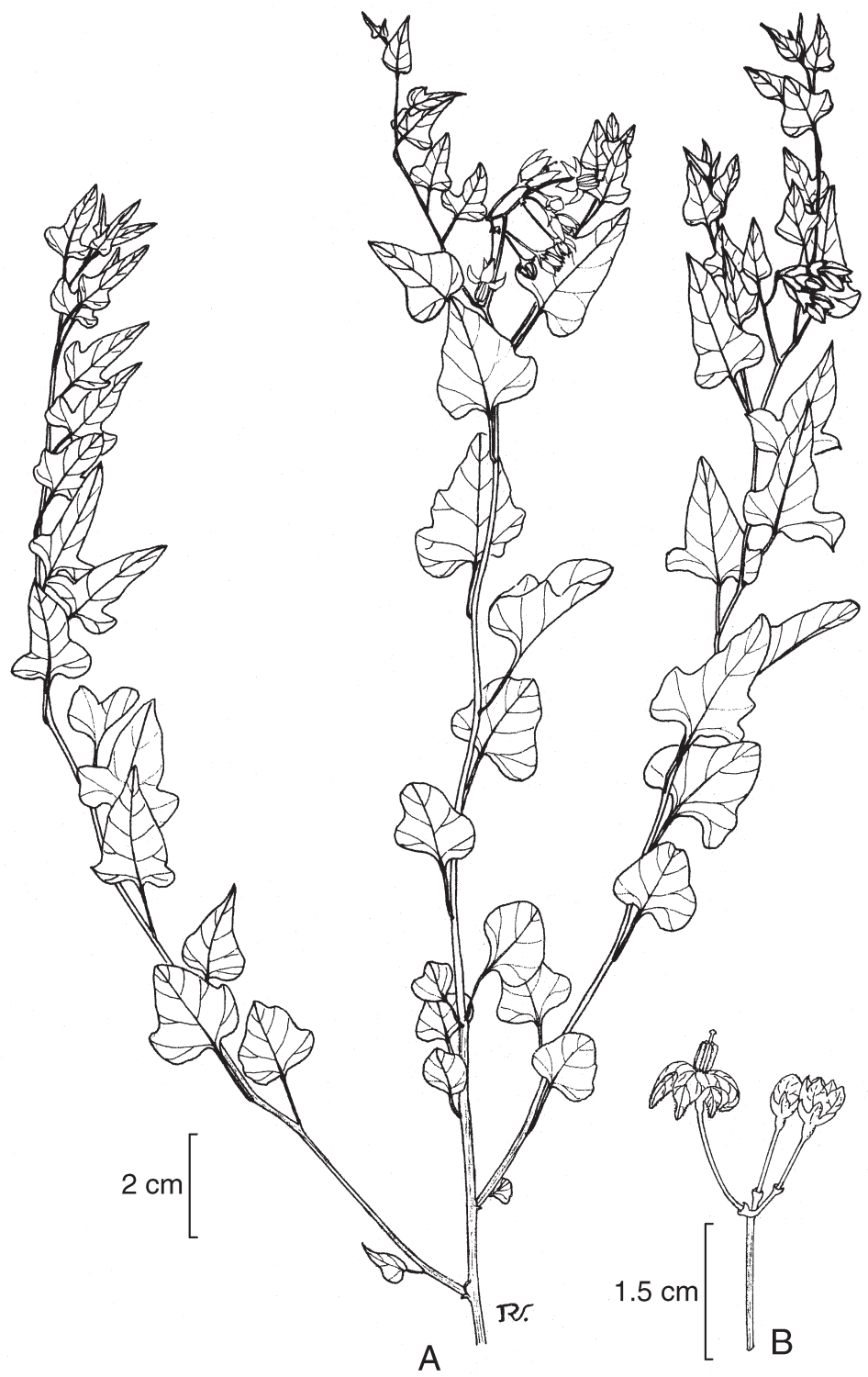

Fig. 1. Solanum triquetrum. A. hábito de la planta; B. detalle de la inflorescencia. (E. Ventura y $E$. López 8564). 
Rodríguez y Vargas: Nuevos Registros de Solanum para el Bajío y Regiones Adyacentes

entonces, el que S. triquetrum tenga esta distribución geográfica. Rzedowski et al. (1996) listaron 264 especies como nuevos registros para la flora de Guanajuato.

Ejemplares revisados: Guanajuato: Mesas del Tigre, municipio de Victoria, E. Ventura y E. López 8564 (IEB); Mangas Cuatas, municipio de Atarjea, E. Ventura y E. López 8953 (IEB). Querétaro: Sótano La Peña, al W de San Antonio Tancoyol, municipio de Jalpan, E. Carranza 2017 (IEB).

\section{AGRADECIMIENTOS}

Los autores agradecen a María del Refugio Vázquez Velasco la elaboración de la ilustración de S. triquetrum y a Roberto González Tamayo la revisión del manuscrito.

\section{LITERATURA CITADA}

Breedlove, D. E. 1986. Listados florísticos de México IV. Flora de Chiapas. Instituto de Biología, Universidad Nacional Autónoma de México. México, D.F. 246 pp.

Correll, D. S. 1952. Section Tuberarium of the genus Solanum of North America and Central America. Agric. Monogr. U.S.D.A. 11: 1-243.

Correll, D. S. 1961. Four new solanums in section Tuberarium. Wrightia 2: 133-141.

Correll, D. S. 1962. The potato and its wild relatives. Contr. Texas Res. Found., Bot. Stud. 4: 1-606.

D'Arcy, W. G. 1991. The Solanaceae since 1976, with a review of its biogeography. In: Hawkes, J. G., R. N. Lester, M. Nee y N. Estrada-R. (eds.). Solanaceae III: taxonomy, chemistry and evolution. The Royal Botanical Garden Kew. Richmond, Surrey. pp. 75-113.

Flores C., R. 1966. Estudio preliminar del género Solanum, sección Tuberarium, subsección Hyperbasarthrum en México. Tesis. Facultad de Ciencias, Universidad Nacional Autónoma de México. México, D.F. 100 pp.

González-Elizondo, M., S. González-Elizondo y Y. Herrera Arrieta. 1991. Listados florísticos de México IX. Flora de Durango. Instituto de Biología, Universidad Nacional Autónoma de México. México, D.F. 195 pp.

Hawkes, J. G. 1990. The potato: evolution, biodiversity and genetic resources. Belhaven Press. Londres. $259 \mathrm{pp}$.

Nee, M. 1993. Solanaceae II. Flora de Veracruz 72: 1-158.

Reeves, R. G. y D. C. Bain. 1947. Flora of South Central Texas. The Exchange Store, A. and M. College of Texas. College Station, Texas. pp. 138-143.

Rodríguez, A. y O. Vargas. 1994. Las especies de papa silvestre (Solanum L. Sección Petota Dumortier) en Jalisco. Bol. Inform. Inst. Bot. Univ. Guadalajara 2 (1-2): 1-68.

Rodríguez-Jiménez, S. y J. Espinosa-Garduño. 1996. Listado florístico del estado de Michoacán sección V (Angiospermae: Najadaceae-Zygophyllaceae). Flora del Bajío y Regiones Adyacentes. Fascículo complementario XV. Instituto de Ecología, A.C., Centro Regional del Bajío. Pátzcuaro, Mich. 344 pp.

Rzedowski, J., G. Calderón de Rzedowski y R. Galván. 1996. Nota sobre la vegetación de la flora del noreste del estado de Guanajuato. Flora del Bajío y de Regiones Adyacentes. Fascículo complementario XIV. Instituto de Ecología, A.C., Centro Regional del Bajío. Pátzcuaro, Mich. $22 \mathrm{pp}$. 
Rzedowski, J. y G. Calderón de Rzedowski. 1991. Guía para los autores y normas editoriales. Flora del Bajío y de Regiones Adyacentes. Fascículo complementario I. Instituto de Ecología, A.C., Centro Regional del Bajío. Pátzcuaro, Mich. 14 pp.

Standley, P. C. 1924. Trees and shrubs of Mexico. Contr. U.S. Natl. Herb. 23: 1-1721.

Recibido en marzo de 2001. Aceptado en septiembre de 2001. 